\title{
Metabotyping and its application in targeted nutrition: an overview
}

\author{
Anna Riedl ${ }^{1,2 *}$, Christian Gieger ${ }^{1,2,3}$, Hans Hauner ${ }^{4,5,6,7}$, Hannelore Daniel $^{7}$ and Jakob Linseisen ${ }^{1,2,5,8}$ \\ ${ }^{1}$ Helmboltz Zentrum München, German Research Center for Environmental Health (GmbH), Institute of Epidemiology II, \\ Ingolstädter Landstr. 1, 85764 Neuberberg, Germany \\ ${ }^{2}$ German Center for Diabetes Research (DZD e.V.), Ingolstädter Landstr. 1, 85764 Neuherberg, Germany \\ ${ }^{3}$ Research Unit of Molecular Epidemiology, Helmboltz Zentrum München, German Research Center for Environmental Health \\ (GmbH), Ingolstädter Landstr. 1, 85764 Neuberberg, Germany \\ ${ }^{4}$ Else Kröner-Fresenius Centre for Nutritional Medicine, Technical University Munich, Gregor-Mendel-Str. 2, 85354 \\ Freising-Weihenstephan, Germany \\ ${ }^{5}$ ZIEL - Institute for Food and Health, Technical University of Munich, Weihenstephaner Berg 1, 85354 Freising, Germany \\ ${ }^{6}$ Klinikum rechts der Isar, Institute of Nutritional Medicine, Technical University of Munich, Uptown München Campus D, \\ Georg-Brauchle-Ring 60/62, 80992 Munich, Germany \\ ${ }^{7}$ Technical University of Munich, Gregor-Mendel-Str. 2, 85354 Freising-Weihenstephan, Germany \\ ${ }^{8}$ Ludwig-Maximilians-Universität München, Universitäres Zentrum für Gesundheitswissenschaften am Klinikum Augsburg \\ (UNIKA-T), Neusässer Str. 47, 86156 Augsburg, Germany
}

(Submitted 21 December 2016 - Final revision received 31 March 2017 - Accepted 8 June 2017 - First published online 19 July 2017)

\section{Abstract}

Metabolic diversity leads to differences in nutrient requirements and responses to diet and medication between individuals. Using the concept of metabotyping - that is, grouping metabolically similar individuals - tailored and more efficient recommendations may be achieved. The aim of this study was to review the current literature on metabotyping and to explore its potential for better targeted dietary intervention in subjects with and without metabolic diseases. A comprehensive literature search was performed in PubMed, Google and Google Scholar to find relevant articles on metabotyping in humans including healthy individuals, population-based samples and patients with chronic metabolic diseases. A total of thirty-four research articles on human studies were identified, which established more homogeneous subgroups of individuals using statistical methods for analysing metabolic data. Differences between studies were found with respect to the samples/ populations studied, the clustering variables used, the statistical methods applied and the metabotypes defined. According to the number and type of the selected clustering variables, the definitions of metabotypes differed substantially; they ranged between general fasting metabotypes, more specific fasting parameter subgroups like plasma lipoprotein or fatty acid clusters and response groups to defined meal challenges or dietary interventions. This demonstrates that the term 'metabotype' has a subjective usage, calling for a formalised definition. In conclusion, this literature review shows that metabotyping can help identify subgroups of individuals responding differently to defined nutritional interventions. Targeted recommendations may be given at such metabotype group levels. Future studies should develop and validate definitions of generally valid metabotypes by exploiting the increasingly available metabolomics data sets.

Key words: Metabotypes: Metabotyping: Metabolic phenotypes: Targeted nutrition: enable Cluster

The human metabolome is influenced by genetic, transcriptional and post-transcriptional factors as well as by the gut microbiome and environmental factors like diet and other lifestyle determinants $^{(1,2)}$. It is well known that individuals show large differences in their nutrient requirements and responses to diet and medication according to their metabolic characteristics ${ }^{(2-5)}$. Specific dietary recommendations or drug treatments for disease states should thus be tailored to optimise the benefit to the individual. Equally important, specific treatments should not be provided to individuals with only a minor response or a lack of positive response to the intervention. The concept of personalisation is supposed to be more effective with respect to individual benefit:risk ratio and health-care costs than currently used general dietary recommendations and standard treatments for chronic disease ${ }^{(3-8)}$.

Such efforts have led to the concept of metabotyping or metabolic phenotyping, which describes the categorisation of individuals based on their metabolic or phenotypic characteristics into more homogeneous subgroups, the so-called metabotypes or metabolic phenotypes. This concept implies that individuals within a subgroup show a high metabolic similarity and those in different subgroups show a high dissimilarity. Metabotyping could, thus, allow the identification of subpopulations or specific patient groups responding differently to a defined dietary or medical

* Corresponding author: A. Riedl, fax +49 893187 2951, email anna.riedl@helmholtz-muenchen.de 
intervention, promising better nutritional and medical treatment at the metabotype group level ${ }^{(6,9-13)}$.

The metabotyping approach has been used widely in healthy animals ${ }^{(14,15)}$ as well as in rodent models of disease for testing drug effects ${ }^{(16,17)}$. On this basis, it was possible to separate strain-specific metabolic phenotypes or strain subtypes based on the plasma, urine or faecal metabolic profiles, thereby finding diagnostic and prognostic biomarker differences between groups $^{(14-26)}$. Strain subtypes could be established by sex ${ }^{(19,23-25)}$, age $^{(22)}$ diet $^{(20,26)}$ or diurnal time of sample collection ${ }^{(18,21,25)}$

Further, several human studies have been conducted to define specific metabotypes, but these studies used a variety of methods and inconsistent definitions, indicating that the term 'metabotype' is often used with quite a different meaning. In reviews on personalised nutrition, O'Donovan et al. ${ }^{(6)}$ and Brennan ${ }^{(13)}$ proposed the concept of metabotyping and provided examples of articles using the metabotyping approach.

The aim of this paper was to review the existing literature on metabotyping in human studies, to show its application in targeted nutrition and, thus, to provide recommendations for future studies in this field.

\section{Methods}

A comprehensive literature search was performed using PubMed, Google and Google Scholar up to May 2016. However, this is not a strictly systematic review as described, for example, by the Cochrane Collaboration ${ }^{(27)}$ because of many open questions. The first search strategy addressed the definition of metabotypes in healthy individuals or population-based samples to find evidence for differences in metabolism and corresponding subgroups. The second search was conducted on the definition of metabotypes in patients with chronic diet-related metabolic diseases (obesity, metabolic syndrome, diabetes, dyslipidaemia, hyperlipidaemia, hyperuricemia, gout and hypertension) for diagnosing or establishing metabolically homogeneous patient subgroups.

Different combinations of the following keywords were used to search for studies that performed metabotyping in healthy subjects or in population-based samples: 'metabotype', 'metabolic phenotype', 'metabolomic phenotype', 'molecular phenotype', 'clinical phenotype', 'biochemical phenotype', 'metabolic profile', 'metabolomic profile', 'metabolic pattern', 'nutritional phenotype', 'nutritype', 'metabolome', 'metabolomics', 'metabolism' or 'metabolic response' and 'cluster', 'pattern', 'subgroup', 'subtype', 'cluster analysis' or 'principal component analysis'. In addition, an extended search was conducted on this topic including information on underlying causes for differences in metabolism between individuals, namely with regard to genetics, epigenetics, transcriptomics or the microbiome ${ }^{(5)}$. To this end, the search terms 'genetics', 'genotype', 'SNP', 'epigenetics', 'transcriptomics', 'gut microbiota' or 'enterotype' were added to the search strategy mentioned above.

The literature search concerning the definition of metabotypes in patients was restricted to frequent chronic metabolic diseases with a strong relation to diet. This selection was based on the worldwide growing prevalence of diet-related metabolic diseases such as obesity and type 2 diabetes, on the one hand, and on the fact that, besides tailored medical treatments, targeted dietary intervention could also have an important effect on diet-related diseases, on the other ${ }^{(28)}$. Thus, in addition to the keywords mentioned above concerning the definition of metabotypes in healthy subjects or population-based samples, the following search terms referring to common metabolic diseases were included in the search strategy: 'obesity', 'adiposity', 'metabolic syndrome', 'diabetes', 'dyslipidaemia', 'hyperlipidaemia', 'hyperuricemia', 'gout' or 'hypertension'. Again, extended searches with keywords addressing underlying causes of metabolic differences were performed.

Relevant articles were selected by first checking titles and abstracts and subsequently the full text of the search results in accordance with the inclusion criteria. Additional studies were identified through supplementary screening of the reference lists of all articles analysed.

The following inclusion and exclusion criteria were used in the literature search: original research articles in English language on human studies, which established homogeneous groups of individuals using statistical analyses based on metabolic data from the body fluids blood and urine. Studies using exclusively other information like genetic, epigenetic, transcriptomic, microbiome, anthropometric or lifestyle data for group establishment were excluded, except in combination with metabolic and/or metabolomics data. In addition, studies in which metabotyping was based only on the combination of simple cut-off points of metabolic variables instead of on statistical analyses, as in the definition of the metabolic syndrome, were not included in this review. In general, all types of study designs were accepted and there were no restrictions on sample size. However, the study populations were limited to healthy subjects or population-based samples in the first search and - for the definition of patient subgroups - to individuals affected by common chronic metabolic diseases in the second search. Extreme or rare chronic diet-related metabolic diseases were not included.

\section{Results}

In total, thirty-four articles met the inclusion criteria, of which twenty-five articles were related to the definition of metabotypes in healthy subjects or population-based samples, and nine articles were related to the definition of patient subgroups with common metabolic diseases revealed by metabotyping.

\section{Definition of metabotypes in healthy subjects or population-based samples}

Tables 1 and 2 summarise the key features of the twenty-five articles identified according to the definition of metabotypes in healthy subjects or population-based samples. Table 1 gives an overview of twenty articles defining metabotypes based on fasting data. Table 2 shows an additional five articles defining metabotypes on the basis of metabolic response data for different dietary interventions. Both tables present the respective study objectives, designs and samples, the variables for clustering and their preprocessing, the clustering methods used and their validation as well as the main findings. With the exception of four 
Table 1. Definition of metabotypes based on metabolic data in the fasting state

\begin{tabular}{|c|c|c|c|c|c|c|c|}
\hline References & Objective & $\begin{array}{l}\text { Study design and } \\
\text { study sample }\end{array}$ & Variables for clustering & $\begin{array}{l}\text { Preprocessing of } \\
\text { variables }\end{array}$ & Clustering method & Validation of cluster solutions & Main findings \\
\hline $\begin{array}{l}\text { Van Bochove } \\
\text { et al. }\end{array}$ & $\begin{array}{l}\text { Plasma lipoprotein } \\
\text { clusters }\end{array}$ & $\begin{array}{l}\text { Genetics of Lipid- } \\
\text { Lowering Drugs and } \\
\text { Diet Network } \\
\text { (GOLDN) study } \\
(n 775) \text { in the USA }\end{array}$ & $\begin{array}{l}\text { NMR plasma lipoprotein profiles of ten } \\
\text { particles: three VLDL (large, medium } \\
\text { and small), four LDL (IDL, large, } \\
\text { medium small and very small) and three } \\
\text { HDL (large, medium and small) } \\
\text { particles }\end{array}$ & $\begin{array}{l}\text { Normalisation by } \\
\text { standard } \\
\text { deviation }\end{array}$ & $\begin{array}{l}\text { k-Means cluster } \\
\text { analysis (squared } \\
\text { Euclidean distance) }\end{array}$ & $\begin{array}{l}\text { Well-differentiated lipoprotein profiles } \\
\text { by discriminatory variables ( } t \text { test) } \\
\text { Stability of cluster results (500 } \\
\text { replications of clustering to select the } \\
\text { result with the lowest total sum of } \\
\text { distances) } \\
\text { Biologically meaningful groups (Particle } \\
\text { Profiler model) }\end{array}$ & $\begin{array}{l}\text { Three distinct subgroups with } \\
\text { differences in lipid characteristics } \\
\text { (low, medium and high degree of } \\
\text { dyslipidaemia) and in Prevalence of } \\
\text { cardiovascular risk factors } \\
\text { Positive lipid response of two subgroups } \\
\text { (medium and high degree of } \\
\text { dyslipidaemia) to fenofibrate therapy; } \\
\text { the resulting group is larger than groups } \\
\text { based on standard cut-off points for TAG } \\
\text { and HDL }\end{array}$ \\
\hline $\begin{array}{l}\text { O'Sullivan } \\
\text { et al. }{ }^{(30)}\end{array}$ & $\begin{array}{l}\text { Metabolic } \\
\text { phenotypes }\end{array}$ & $\begin{array}{l}\text { Intervention study } \\
\text { ( } n \text { 135 healthy } \\
\text { subjects) of } \\
\text { participants aged } \\
18-63 \text { years in } \\
\text { Ireland }\end{array}$ & $\begin{array}{l}\text { Thirteen blood }{ }^{1} \mathrm{H} \text { NMR biochemical } \\
\text { markers of the metabolic syndrome } \\
\text { (leptin, resistin, adiponectin, IL-6, CRP, } \\
\text { TNF-a, insulin, C-peptide, cholesterol, } \\
\text { TAG, NEFA, glucose, HOMA) and } 25 \\
\text { (OH)D concentrations }\end{array}$ & $z$-Standardisation & $\begin{array}{l}\text { k-Means cluster } \\
\text { analysis (Euclidean } \\
\text { distance) }\end{array}$ & $\begin{array}{l}\text { Well-differentiated metabotypes by } \\
\text { discriminatory variables (ANOVA, } \\
\text { GLM analysis, Bonferroni post hoc } \\
\text { multiple comparison test, PLS-DA } \\
\text { with } R^{2}, Q^{2} \text { and variable importance } \\
\text { in the projection value) } \\
\text { Stability of cluster results (ten } \\
\text { iterations, } 5 \text {-fold cross-validation) } \\
\text { Biologically meaningful groups }\end{array}$ & $\begin{array}{l}\text { Five subgroups with distinct biochemical } \\
\text { profiles } \\
\text { One subgroup with lower serum } 25(\mathrm{OH}) \mathrm{D} \\
\text { and higher levels of adipokines and } \\
\text { resistin (cluster } 5 \text { ) responsive to vitamin- } \\
\text { D supplementation concerning markers } \\
\text { of the metabolic syndrome }\end{array}$ \\
\hline $\begin{array}{l}\text { O'Donovan } \\
\text { et al. }\end{array}$ & $\begin{array}{l}\text { Metabolic } \\
\text { phenotypes }\end{array}$ & $\begin{array}{l}\text { National Adult Nutrition } \\
\text { Survey (NANS) } \\
\text { (n } 896 \text { adults) aged } \\
18-90 \text { years in } \\
\text { Ireland }\end{array}$ & $\begin{array}{l}\text { Four routinely measured and widely } \\
\text { applicable serum markers of metabolic } \\
\text { health (TAG, total cholesterol, direct } \\
\text { HDL-cholesterol and glucose) }\end{array}$ & $\begin{array}{l}z \text {-Standardisation } \\
\text { Outlier exclusion }\end{array}$ & $\begin{array}{l}\text { Two-step cluster } \\
\text { analysis with } \\
k \text {-means cluster } \\
\text { analysis }\end{array}$ & $\begin{array}{l}\text { Well-differentiated groups by } \\
\text { discriminatory variables (GLM } \\
\text { analysis, Bonferroni post hoc test) } \\
\text { Stability of cluster results (two-step } \\
\text { cluster analysis) } \\
\text { Biologically meaningful groups } \\
\end{array}$ & $\begin{array}{l}\text { Three distinct subgroups } \\
\text { Identification of a risk cluster with high } \\
\text { fasting levels of TAG, total cholesterol } \\
\text { and glucose } \\
\text { Development and validation of a decision } \\
\text { tree based on biochemical } \\
\text { characteristics, anthropometry and BP } \\
\text { for personalised dietary advice per } \\
\text { cluster }\end{array}$ \\
\hline $\begin{array}{l}\text { Vázquez- } \\
\text { Fresno } \\
\text { et al. }{ }^{(32)}\end{array}$ & $\begin{array}{l}\text { Clinical } \\
\text { phenotypes }\end{array}$ & $\begin{array}{l}\text { Prospective, } \\
\text { randomised, cross- } \\
\text { over and controlled } \\
\text { study ( } n 57 \\
\text { cardiovascular risk } \\
\text { patients aged } \geq 55 \\
\text { years) in Spain }\end{array}$ & $\begin{array}{l}\text { Sixty-nine biochemical (blood, urinary } \\
{ }^{1} \mathrm{H} \text { NMR) and anthropometric } \\
\text { parameters }\end{array}$ & No preprocessing & $\begin{array}{l}\text { k-Means cluster } \\
\text { analysis (Euclidean } \\
\text { distance) }\end{array}$ & $\begin{array}{l}\text { Well-differentiated groups by } \\
\text { discriminatory variables (ANOVA } \\
\text { Kruskal-Wallis test, Tukey's post hoc } \\
\text { multiple comparison test/Mann- } \\
\text { Whitney test, OSC-PLS-DA) } \\
\text { Internal coherence (Dunn analysis), } \\
\text { external homogeneity (Figure of Merit } \\
\text { analysis) } \\
\text { Stability of cluster results (1000 different } \\
\text { random initialisations of clustering, } \\
100 \text { iterations, } 7 \text {-fold internal cross- } \\
\text { validation) } \\
\text { Biologically meaningful groups }\end{array}$ & $\begin{array}{l}\text { Four distinct subgroups } \\
\text { Identification of the two most } \\
\text { discriminant clusters } 3 \text { and } 4 \\
\text { Different responses to red wine } \\
\text { polyphenols of the two subgroups } \\
\text { (cluster } 3 \text { and } 4 \text { ) }\end{array}$ \\
\hline $\begin{array}{l}\text { Frazier-Wood } \\
\text { et al. }{ }^{(33)}\end{array}$ & $\begin{array}{l}\text { Plasma lipoprotein } \\
\text { clusters }\end{array}$ & $\begin{array}{l}\text { Genetics of Lipid- } \\
\text { Lowering Drugs and } \\
\text { Diet (GOLDN) study } \\
\text { ( } n 1036 \text { aged } 48 \cdot 8 \\
\text { (sD 16.2) years) in } \\
\text { the USA }\end{array}$ & $\begin{array}{l}\text { Plasma lipoprotein diameters (VLDL, LDL, } \\
\text { HDL) by NMR spectroscopy }\end{array}$ & Standardisation & Latent class analysis & $\begin{array}{l}\text { Well-differentiated groups by } \\
\text { discriminatory variables (mixed } \\
\text { effects models) } \\
\text { Stability of cluster results (good } \\
\text { internal reliability) } \\
\text { Biologically meaningful groups }\end{array}$ & $\begin{array}{l}\text { Eight distinct subgroups with different } \\
\text { plasma lipoprotein diameters } \\
\text { Association of the subgroups with the } \\
\text { metabolic syndrome }\end{array}$ \\
\hline Zubair et al. ${ }^{(34)}$ & $\begin{array}{l}\text { Cardiometabolic } \\
\text { risk patterns }\end{array}$ & $\begin{array}{l}\text { Cebu Longitudinal } \\
\text { Health and Nutrition } \\
\text { Survey (CLHNS) } \\
\text { ( } n 1768 \text { women aged } \\
\text { 36-69 years) in the } \\
\text { Philippines }\end{array}$ & $\begin{array}{l}\text { Eight cardiometabolic biomarkers (TAG, } \\
\text { HDL, LDL, CRP, systolic and diastolic } \\
\text { BP, HOMA-IR and glucose) }\end{array}$ & $z$-Standardisation & $\begin{array}{l}\text { k-means cluster } \\
\text { analysis (Euclidean } \\
\text { distance) }\end{array}$ & $\begin{array}{l}\text { Well-differentiated groups by } \\
\text { discriminatory variables } \\
\text { (multinomial logistic regression) } \\
\text { Stability of cluster results (1000 } \\
\text { iterations, different cluster numbers) } \\
\text { Biologically meaningful groups }\end{array}$ & $\begin{array}{l}\text { Five distinct subgroups of } \\
\text { cardiometabolic risk: 'healthy', 'high } \\
\text { BP', 'low HDL', 'insulin resistant' and } \\
\text { 'high CRP' }\end{array}$ \\
\hline
\end{tabular}


Table 1. Continued

\begin{tabular}{|c|c|c|c|c|c|c|c|}
\hline References & Objective & $\begin{array}{l}\text { Study design and } \\
\text { study sample }\end{array}$ & Variables for clustering & $\begin{array}{l}\text { Preprocessing of } \\
\text { variables }\end{array}$ & Clustering method & Validation of cluster solutions & Main findings \\
\hline Zubair et al. ${ }^{(35)}$ & $\begin{array}{l}\text { Cardiometabolic } \\
\text { risk patterns }\end{array}$ & $\begin{array}{l}\text { Cebu Longitudinal } \\
\text { Health and Nutrition } \\
\text { Survey (CLHNS) } \\
\text { ( } n 1621 \text { individuals } \\
\text { aged } 21 \text { (SE 0.0) } \\
\text { years) in the } \\
\text { Philippines }\end{array}$ & $\begin{array}{l}\text { Eight cardiometabolic biomarkers (TAG, } \\
\text { HDL, LDL, CRP, systolic and diastolic } \\
\text { BP, HOMA-IR and glucose) }\end{array}$ & $z$-Standardisation & $\begin{array}{l}\text { k-Means cluster } \\
\text { analysis }\end{array}$ & $\begin{array}{l}\text { Well-differentiated groups by } \\
\text { discriminatory variables } \\
\text { (multinomial logistic regression) } \\
\text { Stability of cluster results (iterations, } \\
\text { different cluster numbers) } \\
\text { Biologically meaningful groups }\end{array}$ & $\begin{array}{l}\text { Five distinct sex-specific subgroups of } \\
\text { cardiometabolic risk: 'healthy/high } \\
\text { HDL', 'healthy/low BP', 'high BP' } \\
\text { 'insulin resistant/high TAG' and 'high } \\
\text { CRP', } \\
\text { Prediction of clusters by diet, adiposity } \\
\text { and environment }\end{array}$ \\
\hline $\begin{array}{l}\text { Wilcox } \\
\text { et al. }\end{array}$ & $\begin{array}{l}\text { Metabolic } \\
\text { phenotypes }\end{array}$ & $\begin{array}{l}\text { Framingham Heart } \\
\text { Study (FHS) cohort } \\
(n \text { 2885) in the USA }\end{array}$ & CVD risk factors & $\begin{array}{l}\text { Categorisation of } \\
\text { variables } \\
\text { Data reduction by } \\
\text { multiple- } \\
\text { correspondence } \\
\text { analysis }\end{array}$ & $\begin{array}{l}\text { Two-staged clustering: } \\
k \text {-means cluster } \\
\text { analysis and } \\
\text { hierarchical cluster } \\
\text { analysis }\end{array}$ & $\begin{array}{l}\text { Well-differentiated groups by } \\
\text { discriminatory variables (probability } \\
\text { of cluster membership by binary } \\
\text { logistic regression, genome-wide } \\
\text { linkage analyses) } \\
\text { Stability of cluster results (iterations, } \\
\text { two cluster analyses) } \\
\text { Biologically meaningful groups }\end{array}$ & $\begin{array}{l}\text { Four distinct subgroups: one healthy } \\
\text { group, two groups with mild to } \\
\text { moderately elevated lipid levels, and } \\
\text { one group with strongly elevated lipid } \\
\text { levels } \\
\text { Assessment of heritability of traits }\end{array}$ \\
\hline $\begin{array}{l}\text { Wilcox } \\
\text { et al. }\end{array}$ & $\begin{array}{l}\text { Metabolic } \\
\text { phenotypes }\end{array}$ & $\begin{array}{l}\text { Framingham Heart } \\
\text { Study (FHS) } \\
\text { offspring cohort } \\
(n \text { 2760) in the USA }\end{array}$ & CVD risk factors & $\begin{array}{l}\text { Categorisation of } \\
\text { variables } \\
\text { Data reduction by } \\
\text { multiple- } \\
\text { correspondence } \\
\text { analysis }\end{array}$ & $\begin{array}{l}\text { Two-staged clustering: } \\
k \text {-means cluster } \\
\text { analysis and } \\
\text { hierarchical cluster } \\
\text { analysis } \\
\end{array}$ & $\begin{array}{l}\text { Well-differentiated groups by } \\
\text { discriminatory variables (probability } \\
\text { of cluster membership by binary } \\
\text { logistic regression, genome-wide } \\
\text { association analyses) } \\
\text { Stability of cluster results (iterations, } \\
\text { two cluster analyses) } \\
\text { Biologically meaningful groups }\end{array}$ & $\begin{array}{l}\text { Five distinct subgroups: One group } \\
\text { dropped because of missing data, two } \\
\text { healthy groups, one group with } \\
\text { features of the metabolic syndrome } \\
\text { and one group with features of the } \\
\text { metabolic syndrome and obesity } \\
\text { Genetic associations, but loss of } \\
\text { significance after stratification/ } \\
\text { adjustments }\end{array}$ \\
\hline Tzeng et al. ${ }^{(38)}$ & $\begin{array}{l}\text { Metabolic } \\
\text { phenotypes }\end{array}$ & $\begin{array}{l}\text { Study ( } n 573 \text { women of } \\
\text { reproductive age) in } \\
\text { Taiwan }\end{array}$ & $\begin{array}{l}\text { Ten cardiovascular and metabolic risk factors } \\
\text { (systolic and diastolic BP, waist size, } \\
\text { fasting insulin, fasting glucose, 2-h } \\
\text { glucose, cholesterol, TAG, HDL and LDL) }\end{array}$ & No preprocessing & $\begin{array}{l}\text { Hierarchical cluster } \\
\text { analysis (Ward's } \\
\text { method and within- } \\
\text { group linkage) }\end{array}$ & $\begin{array}{l}\text { Well-differentiated groups by } \\
\text { discriminatory variables ( } X^{2} \text { test, } \\
\text { Fisher's exact test, ANOVA, one- } \\
\text { way ANOVA post hoc range } \\
\text { (Dunnett's) test) } \\
\text { Stability of cluster results (two cluster } \\
\text { analyses) } \\
\text { Biologically meaningful groups }\end{array}$ & $\begin{array}{l}\text { Two distinct subgroups (low- and high- } \\
\text { risk group) } \\
\text { Association between endocrine } \\
\text { disturbances and increased risk for } \\
\text { metabolic diseases }\end{array}$ \\
\hline Li et al. ${ }^{(39)}$ & $\begin{array}{l}\text { Plasma fatty acid } \\
\text { patterns }\end{array}$ & $\begin{array}{l}\text { Irish National Adult } \\
\text { Nutrition Survey } \\
\text { (NANS) }(n 1052 \\
\text { aged } 42 \cdot 9 \text { (sD 16.5) } \\
\text { years) in Ireland }\end{array}$ & Twenty-six plasma fatty acids & $\begin{array}{l}\text { Log-transformation } \\
\text { of skewed data } \\
\text { Exclusion of } \\
\text { outliers } \\
\text { Standardisation } \\
\text { (Subtraction of } \\
\text { minimum and } \\
\text { division by } \\
\text { range) }\end{array}$ & $\begin{array}{l}\text { k-Means cluster } \\
\text { analysis (squared } \\
\text { Euclidean distance) }\end{array}$ & $\begin{array}{l}\text { Well-differentiated groups by } \\
\text { discriminatory variables (GLM, } \\
x^{2} \text { test, ANOVA, Bonferroni } \\
\text { correction) } \\
\text { Stability of cluster results (validation } \\
\text { analysis, scree plot examination, } \\
\text { two-step cluster analysis) } \\
\text { Biologically meaningful groups }\end{array}$ & $\begin{array}{l}\text { Four subgroups with distinct fatty acid } \\
\text { profile } \\
\text { Relationship between plasma fatty acid } \\
\text { patterns, dietary intake and } \\
\text { biomarkers of metabolic health } \\
\text { The subgroup (cluster 3) higher in very- } \\
\text { long-chain SFA and lower in a- } \\
\text { linolenic acid was associated with } \\
\text { metabolic health }\end{array}$ \\
\hline $\begin{array}{l}\text { Bermúdez } \\
\text { et al. }{ }^{(40)}\end{array}$ & $\begin{array}{l}\text { Selection of } \\
\text { metabolically } \\
\text { healthy and sick } \\
\text { individuals for } \\
\text { waist } \\
\text { circumference } \\
\text { cut-off point } \\
\text { selection }\end{array}$ & $\begin{array}{l}\text { Maracaibo City } \\
\text { Metabolic Syndrome } \\
\text { Prevalence Study } \\
\text { (MMSPS) ( } n 1902 \\
\text { aged 38.70 } \\
\text { (SD 15.06) years) in } \\
\text { Venezuela }\end{array}$ & $\begin{array}{l}\text { Eleven metabolic variables (mean arterial } \\
\text { pressure, TAG, cholesterol, HDL, } \\
\text { HOMA2-IR, HOMA2- } \beta \text { cell, HOMA2-S, } \\
\text { fasting glucose, non-HDL-C cholesterol, } \\
\text { TAG/HDL-C index and } \\
\text { hs-CRP) }\end{array}$ & $\begin{array}{l}\text { Log-transformation } \\
\text { of skewed data } \\
\text { Classification } \\
\text { according to BMI } \\
\text { before the two- } \\
\text { step cluster } \\
\text { analysis }\end{array}$ & $\begin{array}{l}\text { Two-step cluster } \\
\text { analysis: } \\
\text { hierarchical } \\
\text { (centroid-based) } \\
\text { and } k \text {-means cluster } \\
\text { analysis (Euclidean } \\
\text { distance) }\end{array}$ & $\begin{array}{l}\text { Well-differentiated groups by } \\
\text { discriminatory variables ( } t \text { test, } \\
\text { ANOVA, cohesion, separation, } \\
\text { silhouette coefficient) } \\
\text { Stability of cluster results (training and } \\
\text { validation data set with Cohen's } \\
\kappa \text { coefficient) } \\
\text { Biologically meaningful groups }\end{array}$ & $\begin{array}{l}\text { Six subgroups with distinct } \\
\text { cardiometabolic profiles } \\
\text { Most predictive variables (HOMA2-IR, } \\
\text { HOMA2- } \beta \text { cell and TAG) } \\
\text { Selection of a cut-off point for waist } \\
\text { circumference ( } 91 \mathrm{~cm} \text { for women and } \\
98 \mathrm{~cm} \text { for men) }\end{array}$ \\
\hline Micciolo ${ }^{(41)}$ & $\begin{array}{l}\text { Metabolic } \\
\text { phenotypes }\end{array}$ & $\begin{array}{l}\text { Patients of one general } \\
\text { practice in Castel } \\
\text { D'Azzano }(n 458 \\
\text { aged 21-60 years) in } \\
\text { Italy }\end{array}$ & $\begin{array}{l}\text { Seven metabolic variables (glucose, uric } \\
\text { acid, TAG, cholesterol, LDL and HDL } \\
\text { (both total and percentage)) and BP } \\
\text { levels or nine anthropometric } \\
\text { characteristics (six skinfolds and three } \\
\text { circumferences) }\end{array}$ & $\begin{array}{l}\text { Log-transformation } \\
\text { of skewed data } \\
\text { Standardisation } \\
\text { (subtraction of } \\
\text { mean and } \\
\text { division by } \\
\text { standard } \\
\text { deviation) }\end{array}$ & $\begin{array}{l}\text { k-Means cluster } \\
\text { analysis (separately } \\
\text { on anthropometric } \\
\text { and metabolic } \\
\text { variables for each } \\
\text { sex) }\end{array}$ & $\begin{array}{l}\text { Well-differentiated groups by } \\
\text { discriminatory variables (hierarchical } \\
\text { algorithm for number of clusters, one- } \\
\text { way ANOVA, } x^{2} \text { statistics) } \\
\text { Stability of cluster results (five iterations, } \\
\text { cross-classification of cluster results } \\
\text { using correspondence analysis, } \gamma \\
\text { coefficient and correlation coefficient) } \\
\text { Biologically meaningful groups }\end{array}$ & $\begin{array}{l}\text { Seven distinct subgroups for men and } \\
\text { women, respectively } \\
\text { Solution with anthropometric variables } \\
\text { more stable than solution with } \\
\text { metabolic variables } \\
\text { Significantly different metabolic patterns } \\
\text { with anthropometric and metabolic } \\
\text { variables } \\
\text { Associations between anthropometric } \\
\text { characteristics and metabolic profiles }\end{array}$ \\
\hline
\end{tabular}


Table 1. Continued

\begin{tabular}{|c|c|c|c|c|c|c|c|}
\hline References & Objective & $\begin{array}{l}\text { Study design and } \\
\text { study sample }\end{array}$ & Variables for clustering & $\begin{array}{l}\text { Preprocessing of } \\
\text { variables }\end{array}$ & Clustering method & Validation of cluster solutions & Main findings \\
\hline $\begin{array}{l}\text { Baumgartner } \\
\text { et al. }{ }^{(42)}\end{array}$ & $\begin{array}{l}\text { Cardiovascular risk } \\
\text { factor groups }\end{array}$ & $\begin{array}{l}\text { Cross-sectional study } \\
\text { ( } n \text { 317 individuals } \\
\text { aged 18-88 years) in } \\
\text { the USA }\end{array}$ & $\begin{array}{l}\text { Cardiovascular risk factors (BP, plasma } \\
\text { lipids, lipoprotein cholesterols and } \\
\text { serum glucose) }\end{array}$ & $\begin{array}{l}\text { Log-transformation } \\
\text { of skewed data } \\
\text { Standardisation } \\
\text { (subtraction of } \\
\text { mean and } \\
\text { division by } \\
\text { standard } \\
\text { deviation) }\end{array}$ & $\begin{array}{l}\text { k-Means cluster } \\
\text { analysis (Euclidean } \\
\text { distance) }\end{array}$ & $\begin{array}{l}\text { Well-differentiated groups by } \\
\text { discriminatory variables (PCA for } \\
\text { number of clusters, one-way } \\
\text { ANOVA, } x^{2} \text { test, discriminant } \\
\text { analysis) } \\
\text { Biologically meaningful groups }\end{array}$ & $\begin{array}{l}\text { Four distinct subgroups for men and } \\
\text { women, respectively } \\
\text { Significant association of cluster } \\
\text { membership with indices of adiposity } \\
\text { but not with adipose tissue } \\
\text { distribution }\end{array}$ \\
\hline $\begin{array}{l}\text { Huang } \\
\text { et al. }\end{array}$ & $\begin{array}{l}\text { Metabolic } \\
\text { phenotypes }\end{array}$ & $\begin{array}{l}\text { West Australian Cohort } \\
\text { (Raine) Study } \\
\text { (n 1094 adolescents } \\
\text { aged } 14 \text { years) in } \\
\text { Australia }\end{array}$ & $\begin{array}{l}\text { The Metabolic syndrome components } \\
\text { (TAG, BMI, HOMA, systolic BP) }\end{array}$ & $\begin{array}{l}\text { Log-transformation } \\
\text { of skewed data }\end{array}$ & $\begin{array}{l}\text { Two-step cluster } \\
\text { analysis separately } \\
\text { by sex (log- } \\
\text { likelihood distance) }\end{array}$ & $\begin{array}{l}\text { Well-differentiated groups by } \\
\text { discriminatory variables (one-way } \\
\text { ANOVA) } \\
\text { Biologically meaningful groups }\end{array}$ & $\begin{array}{l}\text { Two distinct subgroups (high-risk and } \\
\text { low-risk cluster of cardiovascular and } \\
\text { metabolic disorders) } \\
\text { Relationships between inflammatory } \\
\text { markers and components of a } \\
\text { metabolic syndrome cluster }\end{array}$ \\
\hline $\begin{array}{l}\text { Andreeva- } \\
\text { Gateva } \\
\text { et al. }\end{array}$ & $\begin{array}{l}\text { Metabolic } \\
\text { phenotypes }\end{array}$ & $\begin{array}{l}\text { Cross-sectional study } \\
\text { ( } n \text { 113 subjects aged } \\
21-70 \text { years with an } \\
\text { increased risk for } \\
\text { type } 2 \text { diabetes) in } \\
\text { Bulgaria }\end{array}$ & $\begin{array}{l}\text { Components of the metabolic syndrome: } \\
\text { anthropomorphic measurements, lipid } \\
\text { and carbohydrate parameters (during } \\
\text { oral glucose-tolerance test), insulin, } \\
\text { C-peptide, creatinine, CRP, liver tests, } \\
\beta \text {-cell function assessment, insulin } \\
\text { sensitivity and insulin resistance }\end{array}$ & $z$-Standardisation & $\begin{array}{l}\text { Hierarchical cluster } \\
\text { analysis (squared } \\
\text { Euclidean distance, } \\
\text { Ward's method) }\end{array}$ & $\begin{array}{l}\text { Well-differentiated groups by } \\
\text { discriminatory variables (test } \\
\text { statistics) } \\
\text { Stability of cluster results (PCA with } \\
\text { Varimax-normalised rotation for } \\
\text { latent factor identification) } \\
\text { Biologically meaningful groups }\end{array}$ & $\begin{array}{l}\text { Two distinct subgroups } \\
\text { Association of clusters with different } \\
\text { patterns and stages of cardiovascular } \\
\text { risk } \rightarrow \text { diversity of metabolic } \\
\text { disorders in subjects with an } \\
\text { increased risk for type } 2 \text { diabetes }\end{array}$ \\
\hline $\begin{array}{l}\text { Ventura } \\
\quad \text { et al. }\end{array}$ & $\begin{array}{l}\text { Risk profiles for the } \\
\text { metabolic } \\
\text { syndrome }\end{array}$ & $\begin{array}{l}\text { Longitudinal study } \\
\text { (non-clinical sample } \\
\text { of } n 154 \text { adolescent } \\
\text { girls aged } 13 \text { years) } \\
\text { in the USA }\end{array}$ & $\begin{array}{l}\text { Six metabolic syndrome factors (systolic } \\
\text { and diastolic BP, HDL, TAG, waist } \\
\text { circumference and blood glucose) }\end{array}$ & Standardisation & $\begin{array}{l}\text { Mixture model (or } \\
\text { latent profile } \\
\text { analysis) }\end{array}$ & $\begin{array}{l}\text { Well-differentiated groups by } \\
\text { discriminatory variables (GLM, } \\
\text { ANOVA, Fisher's least significant } \\
\text { difference comparison, } x^{2} \text { test, } \\
\text { Fisher's exact test) } \\
\text { Stability of cluster results (AIC, BIC, } \\
\text { multiple iterations, different cluster } \\
\text { numbers) } \\
\text { Bioloaically meaninaful aroups }\end{array}$ & $\begin{array}{l}\text { Four distinct subgroups of risk profiles } \\
\text { for the metabolic syndrome } \\
\text { Differences in developmental, lifestyle } \\
\text { and family history variables between } \\
\text { the subgroups }\end{array}$ \\
\hline Bucci et al. ${ }^{(46)}$ & $\begin{array}{l}\text { Cardiovascular risk } \\
\text { phenotypes }\end{array}$ & $\begin{array}{l}\text { Data sets from France } \\
\text { of the Pôle } \\
\text { Cardiovasculaire de } \\
\text { l'Hôpital Européen } \\
\text { Georges-Pompidou } \\
(n \text { 618) and from } \\
\text { Uruguay }(n 123)\end{array}$ & $\begin{array}{l}\text { Five clinical variables (age, systolic and } \\
\text { diastolic BP, LDL and HDL) }\end{array}$ & No preprocessing & $\begin{array}{l}\text { k-Means cluster } \\
\text { analysis (Euclidean } \\
\text { distance) }\end{array}$ & $\begin{array}{l}\text { Validation using Framingham index } \\
\text { Well-differentiated groups by } \\
\text { discriminatory variables ( } t \text { test) } \\
\text { Stability of cluster results (iterations, } \\
\text { silhouette index) } \\
\text { Biologically meaningful groups }\end{array}$ & $\begin{array}{l}\text { Two distinct subgroups in the data sets } \\
\text { of France and Uruguay, respectively } \\
\text { Association of clusters with } \\
\text { cardiovascular risk patterns }\end{array}$ \\
\hline $\begin{array}{l}\text { Moazzami } \\
\text { et al. }{ }^{(47)}\end{array}$ & $\begin{array}{l}\text { Metabolic } \\
\text { phenotypes }\end{array}$ & $\begin{array}{l}\text { Randomised, controlled, } \\
\text { cross-over meal study } \\
\text { ( } n \text { 19 postmenopausal } \\
\text { women aged } 61 \\
\text { (sD } 4.8) \text { years) in } \\
\text { Finland }\end{array}$ & $\begin{array}{l}189 \text { metabolites from LC-MS } \\
\text { metabolomics analysis (twenty-one } \\
\text { amino acids, seventeen biogenic } \\
\text { amines, forty-seven acyl-carnitines, } \\
\text { thirty-eight phosphatidylcholines, thirty- } \\
\text { nine acyl-alkyl phosphatidylcholines, } \\
\text { fourteen lysophosphatidylcholines, } \\
\text { fifteen sphingomyelins and one hexose) }\end{array}$ & No preprocessing & $\begin{array}{l}\text { Hierarchical cluster } \\
\text { analysis, O-PLS } \\
\text { and PCA }\end{array}$ & $\begin{array}{l}\text { Well-differentiated groups by } \\
\text { discriminatory variables (O-PLS- } \\
\text { DA, GLM, ANOVA) } \\
\text { Stability of cluster results (three cluster } \\
\text { analyses, cross-validated ANOVA, } \\
\text { constant over three different } \\
\text { sampling days) } \\
\text { Biologically meaningful groups }\end{array}$ & $\begin{array}{l}\text { Two distinct subgroups } \\
\text { Different postprandial metabolic } \\
\text { responses to breads (refined wheat, } \\
\text { whole-meal rye and refined rye } \\
\text { breads) } \rightarrow \text { identification of individuals } \\
\text { with reduced insulin sensitivity } \\
\text { Different metabolic responses after } \\
\text { consumption of different breads }\end{array}$ \\
\hline $\begin{array}{l}\text { Qureshi } \\
\text { et al. } \\
\text { (only) } \\
\text { abstract of a } \\
\text { presentation } \\
\text { available) }\end{array}$ & $\begin{array}{l}\text { Metabolic } \\
\text { phenotypes }\end{array}$ & $\begin{array}{l}\text { Insulin Resistance } \\
\text { Atherosclerosis } \\
\text { Study ( } n 500 \\
\text { individuals) in the } \\
\text { USA }\end{array}$ & $\begin{array}{l}\text { Ninety-three serum metabolites from liquid } \\
\text { chromatography-MS analysis }\end{array}$ & - & $\begin{array}{l}\text { Hierarchical cluster } \\
\text { analysis and PCA }\end{array}$ & $\begin{array}{l}\text { Well-differentiated groups by } \\
\text { discriminatory variables (test } \\
\text { statistics) } \\
\text { Stability of cluster results (different } \\
\text { cluster numbers) } \\
\text { Biologically meaningful groups }\end{array}$ & $\begin{array}{l}133 \text { individuals developed incident } \\
\text { hypertension } \\
\text { Identification of a cluster ( } n \text { 154) with } \\
\text { high risk for incident hypertension } \\
\text { Identification of metabolites associated } \\
\text { with a high risk for incident } \\
\text { hypertension }\end{array}$ \\
\hline
\end{tabular}

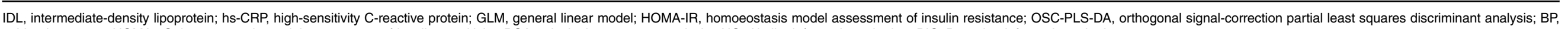
blood pressure; HOMA2-S, homoeostasis model assessment of insulin sensitivity; PCA, principal component analysis; AIC, Akaike information criterion; BIC, Bayesian information criterion. 
Table 2. Definition of metabotypes based on metabolic response data to interventions

\begin{tabular}{|c|c|c|c|c|c|c|c|}
\hline References & Objective & $\begin{array}{l}\text { Study design and } \\
\text { study sample }\end{array}$ & Variables for clustering & Preprocessing of variables & $\begin{array}{l}\text { Clustering } \\
\text { method }\end{array}$ & Validation of cluster solutions & Main findings \\
\hline $\begin{array}{l}\text { Morris } \\
\quad \text { et al. }{ }^{(9)}\end{array}$ & $\begin{array}{l}\text { Response groups to } \\
\text { an oGTT }\end{array}$ & $\begin{array}{l}\text { Metabolic } \\
\text { Challenge } \\
\text { (MECHE) study } \\
\text { ( } n 116 \text { healthy } \\
\text { adults aged } \\
18-60 \text { years) in } \\
\text { Ireland }\end{array}$ & $\begin{array}{l}\text { Response curves of blood } \\
\text { glucose to oGTT (blood } \\
\text { glucose measured during the } \\
\text { OGTT at } 0,10,20,30,60,90 \\
\text { and } 120 \mathrm{~min} \text { ) }\end{array}$ & No preprocessing & $\begin{array}{l}\text { Mixed-model } \\
\text { clustering }\end{array}$ & $\begin{array}{l}\text { Well-differentiated response groups by } \\
\text { discriminatory variables (ANOVA, } \\
\text { GLM, Bonferroni post hoc multiple } \\
\text { comparison test) } \\
\text { Stability of cluster results (oral lipid- } \\
\text { tolerance test) } \\
\text { Biologically meaningful groups }\end{array}$ & $\begin{array}{l}\text { Four distinct subgroups with different } \\
\text { responses to oGTT } \\
\text { One subgroup (cluster 1) as 'at risk' } \\
\text { phenotype having the highest BMI, } \\
\text { TAG, hs-CRP, C-peptide, insulin } \\
\text { and HOMA-IR score and lowest } \\
\mathrm{VO}_{2 \max }\end{array}$ \\
\hline $\begin{array}{l}\text { Krishnan } \\
\quad \text { et al. }{ }^{(49)}\end{array}$ & $\begin{array}{l}\text { Response groups to } \\
\text { meal challenges } \\
\text { with different } \\
\text { glycaemic indices }\end{array}$ & $\begin{array}{l}\text { Cross-over study } \\
\text { ( } n \text { 24 healthy } \\
\text { premenopausal } \\
\text { women aged } \\
20-50 \text { years) in } \\
\text { the USA }\end{array}$ & Blood glucose, insulin and leptin & Range-scaling & PCA & $\begin{array}{l}\text { Well-differentiated response groups by } \\
\text { discriminatory variables (ANOVA, } \\
\text { Tukey's post hoc test, Bonferroni } \\
\text { post hoc multiple comparison test) } \\
\text { Biologically meaningful groups }\end{array}$ & $\begin{array}{l}\text { Three distinct subgroups with different } \\
\text { responses to meal challenges } \\
\text { One subgroup with higher insulin } \\
\text { resistance and another subgroup } \\
\text { with higher leptin values }\end{array}$ \\
\hline $\begin{array}{l}\text { Wang } \\
\text { et al. }{ }^{(50)}\end{array}$ & $\begin{array}{l}\text { Response groups to } \\
\text { dietary carotenoids } \\
\text { in watermelon juice } \\
\text { and tomato juice }\end{array}$ & $\begin{array}{l}\text { Cross-over study } \\
\text { ( } n 23 \text { healthy } \\
\text { subjects) in the } \\
\text { USA }\end{array}$ & $\begin{array}{l}\text { Temporal response of individual } \\
\text { plasma carotenoids } \\
\text { ( } \beta \text {-carotene, lycopene, } \\
\text { phytoene and phytofluene) }\end{array}$ & Normalisation to baseline values & $\begin{array}{l}\text { k-Means cluster } \\
\text { analysis }\end{array}$ & $\begin{array}{l}\text { Well-differentiated response groups by } \\
\text { discriminatory variables ( } t \text { test) } \\
\text { Biologically meaningful groups }\end{array}$ & $\begin{array}{l}\text { Five distinct subgroups with different } \\
\text { plasma responses to dietary } \\
\text { carotenoids } \rightarrow \text { Identification of } \\
\text { strong and weak responders } \\
\text { Response differences between } \\
\text { individual carotenoids and between } \\
\text { interventions } \\
\text { Association of response with genetic } \\
\text { variants of carotenoid-metabolising } \\
\text { enzyme }\end{array}$ \\
\hline $\begin{array}{l}\text { Bouwman } \\
\text { et al. }{ }^{(51)}\end{array}$ & $\begin{array}{l}\text { Response groups to } \\
\text { a 5-week dietary } \\
\text { intervention with } \\
\text { anti-inflammatory } \\
\text { ingredients }\end{array}$ & $\begin{array}{l}\text { Controlled cross- } \\
\text { over study } \\
\text { ( } n 33 \text { men) in } \\
\text { the Netherlands }\end{array}$ & $\begin{array}{l}145 \text { metabolites, seventy-nine } \\
\text { proteins and } 10812 \\
\text { transcripts }\end{array}$ & $\begin{array}{l}\text { Selection of significantly } \\
\text { changed plasma parameters } \\
\text { due to the intervention } \\
\text { Normalisation (subtraction of the } \\
\text { mean and division by the } \\
\text { distance between mean } \\
\text { scores of intervention and } \\
\text { placebo group) }\end{array}$ & $\begin{array}{l}\text { Hierarchical } \\
\text { cluster analysis } \\
\text { (Euclidean } \\
\text { distance, group } \\
\text { average } \\
\text { linkage) }\end{array}$ & $\begin{array}{l}\text { Well-differentiated groups by } \\
\text { discriminatory variables (PLS-DA, } \\
\text { ANOVA) } \\
\text { Stability of cluster results (double cross- } \\
\text { validation of PLS-DA) } \\
\text { Biologically meaningful groups }\end{array}$ & $\begin{array}{l}\text { Two distinct subgroups of inter- } \\
\text { individual responses to intervention } \\
\rightarrow \text { Difference in metabolic stress } \\
\text { profile, inflammatory and oxidative } \\
\text { response } \\
\text { Effects of the nutritional intervention } \\
\text { on oxidative stress, inflammation, } \\
\text { and metabolism } \rightarrow \text { Differentiation } \\
\text { between treated and untreated } \\
\text { individuals }\end{array}$ \\
\hline $\begin{array}{l}\text { Chua } \\
\text { et al. }{ }^{(52)}\end{array}$ & $\begin{array}{l}\text { Circadian metabolic } \\
\text { phenotypes }\end{array}$ & $\begin{array}{l}\text { Study ( } n \text { 20 ethnic- } \\
\text { Chinese male } \\
\text { aged } 21-28 \\
\text { years) in } \\
\text { Singapore }\end{array}$ & $\begin{array}{l}\text { Time course of } 263 \text { plasma } \\
\text { lipids }\end{array}$ & $\begin{array}{l}\text { Iterative feature selection } \\
\text { Elimination of linear trends of } \\
\text { time courses } \\
z \text {-Standardisation }\end{array}$ & $\begin{array}{l}\text { k-Means cluster } \\
\text { analysis and } \\
\text { hierarchical } \\
\text { cluster analysis }\end{array}$ & $\begin{array}{l}\text { Well-differentiated groups by } \\
\text { discriminatory variables (ANOVA, } \\
\text { Kruskal-Wallis test, Bayes method) } \\
\text { Stability of cluster results (consensus } \\
\text { clustering: } 1000 \text { iterations of } k \text {-means } \\
\text { cluster analysis, two cluster methods) } \\
\text { Biologically meaningful groups }\end{array}$ & $\begin{array}{l}\text { Three distinct subgroups } \\
13 \% \text { of lipids showed circadian } \\
\text { variation } \\
\text { Diversity in circadian regulation of } \\
\text { plasma lipids, (glucose and insulin) }\end{array}$ \\
\hline
\end{tabular}

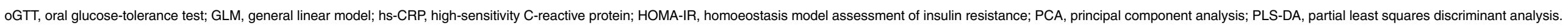


$\operatorname{articles}^{(36,41,42,45)}$, the studies were published within the past decade. The studies were conducted mainly, but not exclusively, in Europe and the USA, either with population-based samples or random samples of healthy individuals. The sample size of the studies varied considerably from twenty to up to 3000 participants. Also, the age range of the study populations differed across the studies with a main focus on adults. Regarding sex, two studies investigated only men ${ }^{(51,52)}$, five studies only women ${ }^{(34,38,45,47,49)}$ and all other studies included both sexes.

For the identification of metabotypes, different numbers of clustering variables were used. Besides the use of full ${ }^{1} \mathrm{H}$ NMR spectra or metabolomics data in some studies ${ }^{(32,47,48,51,52)}$, all other studies used selected metabolites for clustering similar components of the metabolic syndrome ${ }^{(43,45)}$ or cardiovascular risk factors $^{(36,37,42)}$. The type of the cluster variables differed between the studies using blood or urine metabolites, diverse metabolite classes or specifically selected individual metabolite subclasses like lipoproteins or fatty acids and those using fasting metabolites (Table 1) or metabolic responses to dietary interventions (Table 2). According to the number and type of the selected clustering variables, the definitions of metabotypes differed considerably; they ranged between general fasting metabotypes, more specific fasting parameter subgroups like plasma lipoprotein ${ }^{(29,33)}$ or fatty acid clusters $^{(39)}$ and response groups to defined meal challenges or dietary interventions. However, in most studies, at least some standard clinical markers such as glucose, TAG and cholesterol were included. Besides metabolic data, the inclusion of additional phenotypic factors for the definition of metabotypes was implemented in some studies: for example, the consideration of anthropometric parameters like BMI or waist circumference $^{(32,36-38,41,43-45)}$ and blood pressure ${ }^{(34-38,40-43,45,46)}$. However, only the study by Bouwman et al. ${ }^{(51)}$ also assessed some underlying causes for differences in metabolism between subpopulations in the clustering process using transcriptomics data.

Before grouping individuals into metabotypes, diverse preprocessing steps were applied in the studies analysed to the cluster variables such as outlier exclusion, log-transformation of skewed data, dimension reduction (e.g. by multiplecorrespondence analysis) and standardisation (e.g. range-scaling or $z$-standardisation). Different unsupervised learning methods were used in the studies to define relatively homogeneous metabolic groups of individuals. These included $k$-means cluster analysis, hierarchical clustering and combinations of the two, principal component analysis (PCA), latent class analysis ${ }^{(33)}$ and mixed-model clustering ${ }^{(9,45)}$. Then, supervised learning methods, such as partial least squares regression as well as statistical tests like the $t$ test and ANOVA, were used to find discriminatory variables between the established groups. Clustering indices, cross-validation procedures, repetitions with different cluster seeds and cluster numbers as well as different clustering methods were applied to validate the clustering results. Biologically meaningful metabotypes, which were differentiated using discriminatory variables, also confirmed the clustering results. Using the clustering methods, different numbers of metabotypes were found, ranging between two and eight groups. Some studies identified subgroups of individuals with differential response to nutritional interventions; others only described differences between the subgroups, mainly in the fasting state.
The following two studies are examples for the establishment of metabotypes using metabolite profiles obtained in the fasting state and the subsequent investigation of differences in response to dietary interventions between the subgroups. O'Sullivan et al. $^{(30)}$ described metabotypes in an Irish intervention study with 135 healthy individuals aged 18-63 years. After $z$-standardisation, thirteen blood ${ }^{1} \mathrm{H}$ NMR biochemical markers of the metabolic syndrome and serum vitamin-D levels were used in a $k$-means cluster analysis. Five distinct biologically meaningful clusters were found. Among these, one group with lower serum vitamin-D levels and higher levels of adipokines showed a positive response to vitamin-D supplementation on parameters of the metabolic syndrome. The stability of the cluster result was verified using a 5-fold cross-validation method. Second, Vázquez-Fresno et al. ${ }^{(32)}$ investigated fifty-seven subjects at a high cardiovascular risk aged $\geq 55$ years in a randomised and controlled cross-over study. $k$-Means cluster analysis revealed four well-differentiated and biologically meaningful clusters using sixty-nine blood and urine ${ }^{1} \mathrm{H}$ NMR biochemical markers and anthropometric variables identifying red wine polyphenol-responsive metabotypes. In addition to cross-validation, cluster indices like Dunn analysis and Figure of Merit analysis were used.

An example for the definition of metabotypes based on metabolic response data to a dietary intervention is the Irish Metabolic Challenge (MECHE) study, which included 116 participants aged 18-60 years ${ }^{(9)}$. Mixed-model clustering of blood glucose curves revealed four distinct metabotypes with different responses to an oral glucose-tolerance test, of which one group was identified as a high-risk phenotype. The stability of the differentiated clusters was confirmed by another intervention, an oral lipid-tolerance test. Wang et al. $^{(50)}$ described metabotypes in a dietary intervention with carotenoid-rich beverages in a cross-over design based on twenty-three healthy subjects in the USA. In each carotenoid arm, the responses to all plasma carotenoids were analysed individually. $k$-Means cluster analysis revealed five distinct subgroups with different temporal responses. Subsequently, strong and weak responders to individual dietary carotenoids were identified. The different responses were induced by genetic variants of the carotenoidmetabolising enzyme $\beta$-carotene 15,15 '-monooxygenase 1 .

\section{Definition of patient subgroups with metabolic diseases by metabotyping}

Table 3 presents nine publications that were selected during the literature search on the definition of metabotypes in patients with chronic diet-related metabolic diseases for diagnosing or establishing metabolically homogeneous patient subgroups. All articles were published within the last 10 years and, again, a majority of the studies were performed in Europe and the USA with differences in study design, sample size (between fifty and 50000 participants) and the age range of adults. Both sexes were considered in all studies. The articles describe the diagnosis and subgrouping of patients affected by diabetes, obesity, the metabolic syndrome or dyslipidaemia. Here, again, the definitions of patient subgroups varied according to the use of different numbers of metabolic clustering variables. In addition, the types 


\section{N British Journal of Nutrition}

Table 3. Definition of patient subgroups with metabolic diseases by metabotyping

\begin{tabular}{|c|c|c|c|c|c|c|c|}
\hline References & Objective & $\begin{array}{l}\text { Study design and } \\
\text { study sample }\end{array}$ & Variables for clustering & $\begin{array}{l}\text { Preprocessing of } \\
\text { variables }\end{array}$ & Clustering method & Validation of cluster solutions & Main findings \\
\hline Zák et al. ${ }^{(53)}$ & $\begin{array}{l}\text { Diagnosis and } \\
\text { identification of } \\
\text { distinct } \\
\text { phenotypes of } \\
\text { the metabolic } \\
\text { syndrome }\end{array}$ & $\begin{array}{l}\text { Study ( } n 354 \text { individuals } \\
\text { (166 patients with the } \\
\text { metabolic syndrome } \\
\text { and } 188 \text { controls)) in } \\
\text { the Czech Republic }\end{array}$ & $\begin{array}{l}\text { Initially twenty-two but } \\
\text { reduced to six plasma fatty } \\
\text { acids in plasma } \\
\text { phosphatidylcholine } \\
\text { (dihomo- } \gamma \text {-linolenic, } \\
\text { stearic, myristic, DHA, } \\
\text { DPA and linoleic acids) }\end{array}$ & $\begin{array}{l}\text { Examination of extreme } \\
\text { values } \\
\text { Power transformation } \\
\text { for symmetry and } \\
\text { constant variance } \\
\text { Variable reduction by } \\
\text { linear discriminant } \\
\text { analysis with forward } \\
\text { variable selection } \\
\text { using Wilk's } \lambda \\
\text { criterion }\end{array}$ & $\begin{array}{l}\text { Hierarchical cluster } \\
\text { analysis (Ward's } \\
\text { method with } \\
\text { Euclidean } \\
\text { distance) }\end{array}$ & $\begin{array}{l}\text { Well-differentiated individuals } \\
\text { by discriminatory } \\
\text { metabolites ( } t \text { test, } \\
\text { Wilcoxon's test, Benjamin- } \\
\text { Hochberg correction, } \\
\text { ANCOVA adjustments) } \\
\text { Biologically meaningful } \\
\text { groups }\end{array}$ & $\begin{array}{l}\text { Diagnosis of the metabolic } \\
\text { syndrome } \\
\text { Two distinct subgroups of the } \\
\text { metabolic syndrome with } \\
\text { differences in } \\
\text { concentrations of glucose, } \\
\text { NEFA, HOMA-IR and } \\
\text { conjugated dienes in LDL }\end{array}$ \\
\hline Schader ${ }^{(54)}$ & $\begin{array}{l}\text { Subtypes of type } 2 \\
\text { diabetes }\end{array}$ & $\begin{array}{l}\text { GWAS (Framingham Heart } \\
\text { Study (FRAM), MESA } \\
\text { SHARe Study (MESA), } \\
\text { Atherosclerosis Risk in } \\
\text { Communities study } \\
\text { (ARIC)) (13459 study } \\
\text { participants aged } 30-84 \\
\text { years (832 cases during } \\
\text { follow-up for clustering } \\
\text { and } 12066 \text { controls) in } \\
\text { the USA }\end{array}$ & $\begin{array}{l}\text { Ten metabolic and } \\
\text { anthropometric } \\
\text { characteristics before } \\
\text { diagnosis of type } 2 \\
\text { diabetes (sex, BMI, waist: } \\
\text { hip ratio, TAG, HDL, } \\
\text { glucose, insulin, } \\
\text { cholesterol, systolic BP } \\
\text { and diastolic BP) }\end{array}$ & Standardisation & $\begin{array}{l}\text { k-Means cluster } \\
\text { analysis } \\
\text { (Euclidean } \\
\text { distance) }\end{array}$ & $\begin{array}{l}\text { Well-differentiated individuals } \\
\text { by discriminatory } \\
\text { metabolites ( } t \text { test, Cox } \\
\text { proportional hazards } \\
\text { model) } \\
\text { Stability of cluster results } \\
\text { (Calinski method, twenty- } \\
\text { five iterations) } \\
\text { Biologically meaningful } \\
\text { groups }\end{array}$ & $\begin{array}{l}\text { Two distinct subtypes } \\
\text { No statistical significant } \\
\text { differences in genetic risk } \\
\text { factors between the } \\
\text { subtypes }\end{array}$ \\
\hline Amato et al. ${ }^{(56)}$ & $\begin{array}{l}\text { Subtypes of type } 2 \\
\text { diabetes }\end{array}$ & $\begin{array}{l}\text { Cross-sectional study } \\
\text { ( } n \text { 96 patients with type } \\
2 \text { diabetes aged } 62 \cdot 40 \\
\text { (sD 6.36) years } \\
\text { (range }=51-75 \text { years)) } \\
\text { in Italy }\end{array}$ & $\begin{array}{l}\text { Three fasting serum incretins } \\
\text { (GLP-1, GIP and ghrelin) }\end{array}$ & $\begin{array}{l}\text { Log-transformation of } \\
\text { skewed data }\end{array}$ & $\begin{array}{l}\text { Two-step cluster } \\
\text { analysis } \\
\text { (preclustering } \\
\text { and hierarchical } \\
\text { methods, log- } \\
\text { likelihood } \\
\text { distance) }\end{array}$ & $\begin{array}{l}\text { Well-differentiated individuals } \\
\text { by discriminatory } \\
\text { metabolites ( } t \text { test, } x^{2} \text { test, } \\
\text { Fisher's exact test) } \\
\text { Stability of cluster results } \\
\text { (silhouette coefficient) } \\
\text { Biologically meaningful } \\
\text { groups }\end{array}$ & $\begin{array}{l}\text { Two distinct subgroups with } \\
\text { higher levels of glycated } \\
\mathrm{Hb} \text {, glucagon, fasting } \\
\text { glucose and lower levels of } \\
\text { C-peptide in subgroup } 1\end{array}$ \\
\hline Frei et al. ${ }^{(57)}$ & Subtypes of obesity & $\begin{array}{l}\text { Study ( } n 50 \text { patients aged } \\
21-61 \text { years) in Brazil }\end{array}$ & $\begin{array}{l}\text { Blood parameters before and } \\
\text { after the surgery (BMI, } \\
\text { LDL, HDL, VLDL, Hb, } \\
\text { platelets, leucocytes, TAG, } \\
\text { glucose and bilirubin) }\end{array}$ & $z$-standardisation & $\begin{array}{l}\text { Hierarchical cluster } \\
\text { analysis } \\
\text { (Euclidean } \\
\text { distance) }\end{array}$ & $\begin{array}{l}\text { Well-differentiated individuals } \\
\text { by discriminatory } \\
\text { metabolites (ANOVA, } \\
\text { Bonferroni test) } \\
\text { Stability of cluster results } \\
\text { (Calinski-Harabasz, } \\
\text { silhouette index, different } \\
\text { cluster algorithms } \\
\text { (complete linkage, } \\
\text { average linkage, Ward's } \\
\text { method)) } \\
\text { Biologically meaningful } \\
\text { groups }\end{array}$ & $\begin{array}{l}\text { Two distinct subtypes with } \\
\text { differences in indicators of } \\
\text { the metabolic syndrome } \\
\text { (glucose, LDL, VLDL and } \\
\text { TAG) } \\
\text { Identification of patterns that } \\
\text { hinder recovery after the } \\
\text { bariatric surgery }\end{array}$ \\
\hline
\end{tabular}




\section{N British Journal of Nutrition}

Table 3. Continued

\begin{tabular}{|c|c|c|c|c|c|c|c|}
\hline References & Objective & $\begin{array}{l}\text { Study design and } \\
\text { study sample }\end{array}$ & Variables for clustering & $\begin{array}{l}\text { Preprocessing of } \\
\text { variables }\end{array}$ & Clustering method & Validation of cluster solutions & Main findings \\
\hline $\begin{array}{l}\text { Arguelles } \\
\text { et al. }\end{array}$ & $\begin{array}{l}\text { Subtypes of the } \\
\text { metabolic } \\
\text { syndrome }\end{array}$ & $\begin{array}{l}\text { Hispanic Community } \\
\text { Health Study/Study of } \\
\text { Latinos (HCHS/SOL) } \\
\text { ( } n 15825 \text { Hispanics/ } \\
\text { Latinos aged 18-74 } \\
\text { years) in the USA }\end{array}$ & $\begin{array}{l}\text { Metabolic syndrome } \\
\text { components (waist } \\
\text { circumference, systolic } \\
\text { and diastolic BP, HDL, } \\
\text { TAG, glucose, medication } \\
\text { use) }\end{array}$ & $\begin{array}{l}\text { Log-transformation and } \\
\text { multiplication by } 100 \\
\text { were used for } \\
\text { skewed variables }\end{array}$ & $\begin{array}{l}\text { Latent class } \\
\text { analysis } \\
\text { separately by sex }\end{array}$ & $\begin{array}{l}\text { Well-differentiated individuals } \\
\text { by discriminatory } \\
\text { metabolites (logistic } \\
\text { regression) } \\
\text { Stability of cluster results } \\
\text { (different cluster numbers, } \\
\text { AlC, BIC, ABIC, entropy } \\
\text { and posterior probabilities) } \\
\text { Biologically meaningful } \\
\text { groups }\end{array}$ & $\begin{array}{l}\text { Two distinct subgroups for } \\
\text { men and women, } \\
\text { respectively ('metabolic } \\
\text { syndrome' cluster and } \\
\text { 'non-metabolic syndrome' } \\
\text { cluster) } \\
\text { Association of subgroups } \\
\text { with covariates and CVD } \\
\text { No identification of additional } \\
\text { subtypes of the metabolic } \\
\text { syndrome }\end{array}$ \\
\hline Kim et al. ${ }^{(59)}$ & $\begin{array}{l}\text { Subtypes of } \\
\text { prediabetes }\end{array}$ & $\begin{array}{l}\text { Large Cohort (n } 52139 \\
\text { adult Mayo Clinic } \\
\text { patients) in the USA }\end{array}$ & $\begin{array}{l}\text { Diagnoses (obesity, } \\
\text { hyperlipidaemia, } \\
\text { hypertension, renal failure, } \\
\text { various cardiovascular } \\
\text { conditions), vital signs (BP, } \\
\text { pulse), laboratory results } \\
\text { (glucose, lipids), use of } \\
\text { medication (aspirin, } \\
\text { medication for } \\
\text { hypertension and } \\
\text { hypercholesterolaemia) }\end{array}$ & $\begin{array}{l}\text { Binary transformation } \\
\text { of variables }\end{array}$ & $\begin{array}{l}\text { Bisecting divisive } \\
\text { hierarchical } \\
\text { cluster analysis }\end{array}$ & $\begin{array}{l}\text { Well-differentiated individuals } \\
\text { by discriminatory } \\
\text { metabolites } \\
\text { Biologically meaningful } \\
\text { groups }\end{array}$ & $\begin{array}{l}\text { A subgroup with higher and } \\
\text { another subgroup with } \\
\text { lower risk for diabetes than } \\
\text { the general population } \\
\text { Identification of twelve } \\
\text { highest-risk groups (out of } \\
\text { twenty-six clusters) and } \\
\text { their relevant risk factors } \\
\text { Use of clustering as a } \\
\text { diabetes index } \\
\text { outperforming the } \\
\text { Framingham risk score }\end{array}$ \\
\hline $\begin{array}{l}\text { Botelho } \\
\text { et al. }\end{array}$ & $\begin{array}{l}\text { Subgroups of } \\
\text { dyslipidaemia }\end{array}$ & $\begin{array}{l}\text { Patient data bank at the } \\
\text { Dante Pazzanese } \\
\text { Institute of Cardiology } \\
\text { ( } n 57 \text { individuals aged } \\
30-80 \text { years with } \\
\text { dyslipidaemia } \\
\text { controlled by statins) in } \\
\text { Brazil }\end{array}$ & $\begin{array}{l}\text { Four plasma biomarkers of } \\
\text { oxidative stress } \\
\text { (malondialdehyde, ferric } \\
\text { reducing ability power, 2,2- } \\
\text { diphenyl-1-picrylhydrazyl } \\
\text { radical and oxidised-LDL) }\end{array}$ & $\begin{array}{l}\text { Dimension reduction by } \\
\text { PCA }\end{array}$ & $\begin{array}{l}\text { Hierarchical cluster } \\
\text { analysis (Ward's } \\
\text { method, } \\
\text { Euclidean } \\
\text { distance) }\end{array}$ & $\begin{array}{l}\text { Well-differentiated individuals } \\
\text { by discriminatory } \\
\text { metabolites (ANOVA, } \\
\text { Tukey's post hoc test) } \\
\text { Biologically meaningful } \\
\text { groups }\end{array}$ & $\begin{array}{l}\text { Five distinct subgroups } \\
\text { No difference in dietary } \\
\text { pattern between the } \\
\text { subgroups }\end{array}$ \\
\hline
\end{tabular}

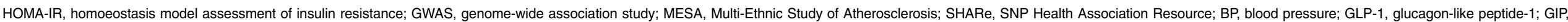
glucose-dependent insulinotropic polypeptide; AIC, Akaike information criterion; BIC, Bayesian information criterion; ABIC, sample size-adjusted BIC; PCA, principal component analysis. 
of clustering variables differed, often depending on the particular disease investigated. For example, Mäkinen et al. ${ }^{(60)}$ used a full blood serum ${ }^{1} \mathrm{H}$ NMR spectrum for the subgrouping of patients with type 1 diabetes. In contrast, Arguelles et al. ${ }^{(58)}$ tried to identify subgroups of the metabolic syndrome using only components of this syndrome (waist circumference, systolic and diastolic blood pressure, HDL, TAG, fasting glucose and medication use) for the clustering procedure. Few studies used additional variables such as anthropometry ${ }^{(54,57,58)}$ or medication use ${ }^{(58,59)}$ along with the metabolic information in the clustering process. As a result, the studies identified different patient subgroups depending on the metabolic data assessed. After the application of various preprocessing steps to the cluster variables as described above, clustering methods like $k$-means cluster analysis, hierarchical clustering and combinations of the two, topological analysis ${ }^{(55)}$, latent class analysis ${ }^{(58)}$ and self-organising maps ${ }^{(60)}$ were applied. Discriminatory variables between the resulting disease subgroups were again identified using test statistics. Moreover, biological meaning, clustering indices, cross-validation procedures, repetitions with different cluster seeds and cluster numbers as well as different clustering algorithms were applied to validate the clustering results. Different numbers of disease subgroups were formed, mainly two to four groups.

An example for the establishment of type 2 diabetes subgroups is the study by Schader ${ }^{(54)}$ using three studies in the USA with a total of 832 patients with type 2 diabetes aged 30-84 years. Applying $k$-means cluster analysis with ten standardised metabolic and anthropometric characteristics assessed before the diagnosis of type 2 diabetes, two subgroups of the disease were found. Despite the stability of the clustering results, measured using the Calinski method and twenty-five repetitions of the clustering method, and strong differentiation of individuals based on discriminatory variables, no statistically significant difference was found between the genetic risk factors among the subgroups. In a smaller sample size of ninety-six patients with type 2 diabetes, Amato et al. ${ }^{(56)}$ used three fasting incretins in a two-step cluster analysis to identify two subgroups of this disease.

\section{Discussion}

This review analysed the literature on metabotyping of individuals in metabolic and nutrition research. In total, thirty-four studies were included in this analysis covering a wide range of populations and using various clustering variables and statistical methods to identify different numbers of metabotypes. Consequently, it is difficult to draw meaningful conclusions regarding the establishment of metabotypes based on these rather heterogeneous studies using different approaches in metabotyping. However, this paper includes all available human studies using metabotyping in healthy subjects, population-based samples and patients with chronic metabolic diseases, and thereby represents the current state of knowledge.

\section{Differences in study populations}

We found a considerable variation in metabotypes across the countries in which the studies were performed, and this could be due to different genetic characteristics, environmental influences (like dietary and cultural behaviour), risk factors and disease rates ${ }^{(5,62-64)}$. This variation was seen to be particularly large between Western countries and East Asian countries, whereas metabotypes across different Western countries displayed substantial overlapping ${ }^{(62,64)}$. As most studies we review here were conducted in Western populations in Europe and the USA, the defined metabotypes seem to be transferable and comparable between these studies. However, there is a lack of data as to whether these metabotypes can be transferred to other ethnic populations.

Comparing metabotypes between different age ranges may be hampered by the physiological ageing process itself, which is characterised by marked changes in metabolism or metabolic flexibility ${ }^{(65)}$. However, it was shown in some studies that the plasma metabotypes (metabolite profiles) of individuals remain relatively stable over a few years ${ }^{(66,67)}$ and only large differences in age seem to be relevant. As many metabolites differ between men and women - for example, steroid hormones or branched chain amino acids ${ }^{(62,68,69)}$ - studies need to consider sex differences. This could be achieved by the exclusion of these sex-specific variables from the clustering process or by separate analyses for men and women.

\section{Differences in variables used for clustering}

The use of diverse types and numbers of clustering variables does not allow a reasonable comparison of the metabotypes identified in different studies. At present, the debate on the most important criteria and variables to be used for the definition of a biologically meaningful metabotype remains open. Equally important, the aim of metabotype definition has to be defined a priori. In 2000, Gavaghan et al. ${ }^{(15)}$ defined a metabotype as 'a probabilistic multiparametric description of an organism in a given physiological state based on analysis of its cell types, biofluids or tissues'. Later, metabotyping was described in several studies as the 'process of grouping similar individuals based on their metabolic or phenotypic characteristics $^{(6,9-13)}$. These wide and general definitions of metabotypes allow the inclusion of all studies establishing subgroups based on (1) healthy or sick people (thus also in the diagnosis or subgrouping of patients), (2) the fasting state or response to interventions, (3) a few or a variety of metabolites and (4) specifically selected single metabolite subclasses like lipoproteins, diverse metabolite subclasses or the addition of other variables like underlying causes for differences in metabolism - for example, genetic, epigenetic or gut microbiome information.

The selection of variables plays an important role in the identification and separation of metabotypes. Grouping of individuals based on a few variables or single specific metabolite classes provides a restricted definition of metabotypes, as only a small part of human metabolism is taken into account. However, for the establishment of plasma lipoprotein clusters in the studies by van Bochove et al. ${ }^{(29)}$ and Frazier-Wood et al. ${ }^{(33)}$, or of plasma fatty acid patterns in the study by Li et al. ${ }^{(39)}$, restriction to the respective lipid variables seemed to be sufficient for subclassification. Likewise, Wang et al $^{(50)}$ considered only the plasma carotenoid levels after a dietary intervention with 
carotenoids. The same was the case in the study by Morris et al. ${ }^{(9)}$ considering only blood glucose levels, measured at several points in time, to identify groups with differential glucose responses to an oral glucose-tolerance test. This is of course in accordance with the current clinical practice for classification of type 2 diabetes based on the plasma kinetics of glucose. In diagnosing or subgrouping patients, the restriction of variables to disease-related parameters could also be sufficient for subclassification. For example, Arguelles et $a l^{(58)}$ established subgroups of the metabolic syndrome patients based on the standard criteria for disease description, namely waist circumference, systolic and diastolic blood pressure, HDL, TAG, fasting glucose and medication use. The grouping in other studies using plasma fatty acids for the description of the metabolic syndrome ${ }^{(53)}$ and fasting incretins for the subgrouping of diabetes ${ }^{(56)}$ could be probably refined by the consideration of additional disease-related variables.

There is no consensus yet on a uniform use of the term 'metabotype', thus it is subjectively applied, usually based on the respective study objectives. In this review, the definitions of metabotypes differed considerably; they ranged between general fasting metabotypes, more specific fasting parameter subgroups like plasma lipoprotein ${ }^{(29,33)}$ or fatty acid clusters ${ }^{(39)}$ and response groups to defined meal challenges or dietary interventions according to the number and type of the selected clustering variables. Although an accepted definition of metabotype seems attractive, there is also the view that there is no need for a strict metabotype definition. On the one hand, it may be argued that a metabotype has by its nature a wide definition and should not be restricted. On the other hand, a better comparability of studies could be achieved using a stricter definition. Even if a strict general definition appears implausible or unrealistic, more precise sub-definitions of metabotypes could be developed, for example for lipid and carbohydrate (glucose) metabolism. Thus, metabolic variables restricted to specific metabolic pathways like to those of lipoproteins may be sufficient depending on the respective study objective.

However, it is assumed that the inclusion of various metabolites originating from different pathways as well as additional information from anthropometry or that obtained by including genetics, epigenetics or the gut microbiome in the process of metabotyping provides a more precise characterisation of individuals and, thus, the establishment of more refined and generally valid metabotypes ${ }^{(70)}$. This can be achieved through the use of '-omics' data such as metabolomics, genomics and epigenomics, where research is growing rapidly ${ }^{(2,71,72)}$. Thus, it may be wise to suggest a stricter definition of generally valid metabotypes in healthy subjects or population-based samples by at least the use of variables originating from different metabolic pathways, preferably the use of targeted or untargeted metabolomics data.

Further, there is no agreement as to whether the definition of metabotypes should be based on fasting data (see Table 1) or rather on metabolic response data to interventions (see Table 2), for which we identified only five studies that met the inclusion criteria. An argument for the use of metabolic response data to interventions is the increase of variation between individuals as some metabolic differences are only visible through challenges and would remain undetected using fasting blood values ${ }^{(73)}$. However, the establishment of metabotypes by means of fasting data allows extensive measurements of larger study populations and is thus more feasible in the general population. It is important to note that intra-individual variations of metabolite concentrations may also occur because of diurnal time, stress, latent diseases as well as by measurement and storage conditions of the samples ${ }^{(5,64,74,75)}$. However, these differences were shown to be smaller than inter-individual differences, suggesting that individual metabotypes are relatively robust ${ }^{(76)}$.

\section{Differences in statistical analyses}

As a variety of statistical methods are available for the establishment of metabotypes ${ }^{(70)}$, there is an on-going discussion on which statistical methods should be used to obtain the best spread between subgroups. The preprocessing of variables is especially dependent on the structure of the variables and the requirements of the subsequent clustering methods. Thus, the implementation of outlier exclusion and data transformation has to be decided individually. If the number of clustering variables exceeds one per ten observations, application of data-reduction analyses like PCA or multiple-correspondence analysis must be considered to avoid over adjustment ${ }^{(77)}$. In many studies included in this review, standardisation has been applied to the cluster variables to avoid bias from different scales and units in the grouping analysis ${ }^{(78,79)}$. The most commonly used method is $z$-standardisation $\left(z=\frac{X-\text { mean }}{\mathrm{SD}}\right)$.

Concerning the different clustering methods ${ }^{(78-82)}, k$-means cluster analysis and hierarchical cluster analysis were applied most commonly. Each clustering method has its own advantages and disadvantages and must be selected depending on the characteristics of the respective data set (e.g. depending on the scale level or the sample size). $k$-Means cluster analysis seems to be more suitable for large data sets than hierarchical clustering. However, the number of clusters has to be specified in advance for $k$-means cluster analysis, whereas hierarchical clustering does not need the number of clusters to be determined $^{(82)}$. In addition, there are novel clustering techniques available in the field of bioinformatics, for example the so-called machine learning methods ${ }^{(83)}$.

The selection of validation criteria like statistical tests and clustering indices is also dependent on the structure of the data. The reproducibility of metabotypes should be tested in a validation data set to confirm the results and to prove their generalisability.

\section{Differences in the main findings}

The aim of most studies was to examine metabolic differences between the established metabotypes and to test associations with certain diseases. However, the application of metabotypes, especially the development of targeted interventions for responsive subgroups, is rather limited in the literature. In addition, intervention by supplementation may increase serum levels in all subgroups but with possibly either larger effects in some subgroups or attainment of a threshold concentration considered to be within the normal range. Thus, responsiveness 
to an intervention does not necessarily mean benefit and, therefore, outcome parameters also need to be properly defined to evaluate the benefit of interventions, which so far has been rare in previous studies. Only few studies investigated the responsiveness of the established metabotypes to dietary interventions with regard to a specific disease. O'Sullivan et al. ${ }^{(30)}$ identified a subgroup with a positive response to vitamin D supplementation concerning the metabolic syndrome; VázquezFresno et $a l .{ }^{(32)}$ detected a subgroup of patients at cardiovascular risk responsive to red wine polyphenols; and Moazzami et al. ${ }^{(47)}$ identified individuals with reduced insulin sensitivity after consumption of bread. There is only one study that developed tailored dietary recommendations for subgroups using a decision-tree approach $^{(31)}$. Until now, the established metabotypes have not been transferred to larger populations for specific, tailored interventions.

\section{Conclusion}

In conclusion, this literature review shows that metabotyping can help identify metabolically similar subpopulations or patient subgroups responding differently to defined nutritional interventions. Consequently, better tailored and, thus, more precise dietary recommendations than generalised advice may be provided to whole populations at a metabotype group level. The aim of future studies should be the refinement of the definition of generally valid metabotypes in large samples, especially with a possibly more precise phenotype description of individuals based on different '-omics' data, particularly metabolomics data. Another aim should be the development of stricter definitions of specific metabotypes for metabolic pathways. The metabotypes should then be tested for differential reactions to diverse dietary factors with regard to properly defined outcome parameters. On the basis of such results, populations can be better stratified in order to provide effective tailored prevention and intervention programs. The implementation of these recommendations in populations may become a future task. Finally, individual health benefits may be improved and the rising costs in the health-care system originating from obesity and other diet-related metabolic diseases may be better controlled.

\section{Acknowledgements}

This study was supported by the enable Cluster and is catalogued by the enable Steering Committee as enable 001 (http://enablecluster.de). This work was funded by a grant from the German Ministry for Education and Research (BMBF) FK 01EA1409E.

Contributions of authors were as follows: A. R. conceived the review, conducted the literature search and wrote the paper; C. G. provided advice regarding the literature search and revised the paper; $H$. H. provided advice regarding the literature search and revised the paper; $H$. D. provided advice regarding the literature search and revised the paper; J. L. conceived the review, provided advice regarding the literature search and revised the paper. All authors have read and approved the final manuscript.

None of the authors has any conflicts of interest to declare.

\section{References}

1. Nicholson JK (2006) Global systems biology, personalized medicine and molecular epidemiology. Mol Syst Biol 2, 52.

2. Beger RD, Dunn W, Schmidt MA, et al. (2016) Metabolomics enables precision medicine: 'A White Paper, Community Perspective'. Metabolomics 12, 149.

3. de Roos B (2013) Personalised nutrition: ready for practice? Proc Nutr Soc 72, 48-52.

4. Nicholson JK \& Holmes E (2006) Global systems biology and personalized healthcare solutions. Discov Med 6, 63-70.

5. Holmes E, Wilson ID \& Nicholson JK (2008) Metabolic phenotyping in health and disease. Cell 134, 714-717.

6. O'Donovan CB, Walsh MC, Gibney MJ, et al. (2016) Can metabotyping help deliver the promise of personalised nutrition? Proc Nutr Soc 75, 106-114.

7. Celis-Morales C, Livingstone KM, Marsaux CF, et al. (2017) Effect of personalized nutrition on health-related behaviour change: evidence from the Food4me European randomized controlled trial. Int J Epidemiol 46, 578-588.

8. Livingstone KM, Celis-Morales C, Navas-Carretero S, et al. (2016) Effect of an Internet-based, personalized nutrition randomized trial on dietary changes associated with the Mediterranean diet: the Food4Me Study. Am J Clin Nutr 104, 288-297.

9. Morris C, O'Grada C, Ryan M, et al. (2013) Identification of differential responses to an oral glucose tolerance test in healthy adults. PLOS ONE $\mathbf{8}$, e 72890 .

10. Nicholson JK, Holmes E, Kinross JM, et al. (2012) Metabolic phenotyping in clinical and surgical environments. Nature 491, 384-392.

11. Kaput J (2008) Nutrigenomics research for personalized nutrition and medicine. Curr Opin Biotechnol 19, 110-120.

12. Brennan L (2008) Session 2: personalised nutrition. Metabolomic applications in nutritional research. Proc Nutr Soc 67, 404-408.

13. Brennan L (2016) Use of metabotyping for optimal nutrition. Curr Opin Biotechnol 44, 35-38.

14. Pontoizeau C, Fearnside JF, Navratil V, et al. (2011) Broadranging natural metabotype variation drives physiological plasticity in healthy control inbred rat strains. J Proteome Res 10, 1675-1689.

15. Gavaghan CL, Holmes E, Lenz E, et al. (2000) An NMR-based metabonomic approach to investigate the biochemical consequences of genetic strain differences: application to the C57BL10J and Alpk:ApfCD mouse. FEBS Lett $\mathbf{4 8 4}$, 169-174.

16. Clayton TA, Lindon JC, Cloarec O, et al. (2006) Pharmacometabonomic phenotyping and personalized drug treatment. Nature 440, 1073-1077.

17. Holmes E, Nicholls AW, Lindon JC, et al. (2000) Chemometric models for toxicity classification based on NMR spectra of biofluids. Chem Res Toxicol 13, 471-478.

18. Gavaghan CL, Wilson ID \& Nicholson JK (2002) Physiological variation in metabolic phenotyping and functional genomic studies: use of orthogonal signal correction and PLS-DA. FEBS Lett 530, 191-196.

19. Gavaghan McKee CL, Wilson ID \& Nicholson JK (2006) Metabolic phenotyping of nude and normal (Alpk:ApfCD, C57BL10J) mice. J Proteome Res 5, 378-384.

20. Fearnside JF, Dumas ME, Rothwell AR, et al. (2008) Phylometabonomic patterns of adaptation to high fat diet feeding in inbred mice. PLOS ONE 3, e1668.

21. Bollard ME, Holmes E, Lindon JC, et al. (2001) Investigations into biochemical changes due to diurnal variation and estrus cycle in female rats using high-resolution (1)H NMR spectroscopy of urine and pattern recognition. Anal Biochem 295, 194-202. 
22. Calvani R, Brasili E, Praticò G, et al. (2014) Fecal and urinary NMR-based metabolomics unveil an aging signature in mice. Exp Gerontol 49, 5-11.

23. Garcia-Perez I, Villasenor A, Wijeyesekera A, et al. (2012) Urinary metabolic phenotyping the slc26a6 (chloride-oxalate exchanger) null mouse model. J Proteome Res 11, 4425-4435.

24. Qiao Q, Li T, Sun J, et al. (2011) Metabolomic analysis of normal (C57BL/6J, 129S1/SvImJ) mice by gas chromatographymass spectrometry: detection of strain and gender differences. Talanta 85, 718-724.

25. Plumb R, Granger J, Stumpf C, et al. (2003) Metabonomic analysis of mouse urine by liquid-chromatography-time of flight mass spectrometry (LC-TOFMS): detection of strain, diurnal and gender differences. Analyst 128, 819-823.

26. Yang Y, Liu Y, Zheng L, et al. (2014) Serum metabonomic analysis of apoE(-/-) mice reveals progression axes for atherosclerosis based on NMR spectroscopy. Mol Biosyst 10, 3170-3178.

27. JPT Higgins \& Green S (editors) (2011) Cochrane Handbook for Systematic Reviews of Interventions Version 5.1.0. The Cochrane Collaboration. http://handbook.cochrane.org/ (accessed November 2016).

28. World Health Organization (2003) Diet, Nutrition and the Prevention of Chronic Diseases. Joint WHO/FAO Expert Consultation. WHO Technical Report Series, no. 916. Geneva: WHO.

29. van Bochove K, van Schalkwijk DB, Parnell LD, et al. (2012) Clustering by plasma lipoprotein profile reveals two distinct subgroups with positive lipid response to fenofibrate therapy. PLOS ONE 7, e38072.

30. O'Sullivan A, Gibney MJ, Connor AO, et al. (2011) Biochemical and metabolomic phenotyping in the identification of a vitamin $\mathrm{D}$ responsive metabotype for markers of the metabolic syndrome. Mol Nutr Food Res 55, 679-690.

31. O'Donovan CB, Walsh MC, Nugent AP, et al. (2015) Use of metabotyping for the delivery of personalised nutrition. $\mathrm{Mol}$ Nutr Food Res 59, 377-385.

32. Vázquez-Fresno R, Llorach R, Perera A, et al. (2016) Clinical phenotype clustering in cardiovascular risk patients for the identification of responsive metabotypes after red wine polyphenol intake. J Nutr Biochem 28, 114-120.

33. Frazier-Wood AC, Glasser S, Garvey WT, et al. (2011) A clustering analysis of lipoprotein diameters in the metabolic syndrome. Lipids Health Dis 10, 237.

34. Zubair N, Kuzawa CW, McDade TW, et al. (2012) Cluster analysis reveals important determinants of cardiometabolic risk patterns in Filipino women. Asia Pac J Clin Nutr 21, 271-281.

35. Zubair N, Kuzawa CW, Lee NR, et al. (2014) Clustering and determinants of cardiometabolic risk factors among Filipino young adults. Asia Pac J Clin Nutr 23, 148-158.

36. Wilcox MA, Wyszynski DF, Panhuysen CI, et al. (2003) Empirically derived phenotypic subgroups - qualitative and quantitative trait analyses. BMC Genet $\mathbf{4}$, Suppl. 1, S15

37. Wilcox M, Li Q, Sun Y, et al. (2009) Genome-wide association study for empirically derived metabolic phenotypes in the Framingham Heart Study offspring cohort. BMC Proc 3, Suppl. 7, S53.

38. Tzeng CR, Chang YC, Chang YC, et al. (2014) Cluster analysis of cardiovascular and metabolic risk factors in women of reproductive age. Fertil Steril 101, 1404-1410.

39. Li K, Brennan L, McNulty BA, et al. (2016) Plasma fatty acid patterns reflect dietary habits and metabolic health: a crosssectional study. Mol Nutr Food Res 60, 2043-2052.

40. Bermúdez V, Rojas J, Salazar J, et al. (2015) Sensitivity and specificity improvement in abdominal obesity diagnosis using cluster analysis during waist circumference cut-off point selection. J Diabetes Res 2015, 750265.
41. Micciolo R (1992) Clustering of hemodynamic and metabolic abnormalities and of anthropometric characteristics in men and women, aged 21-60 years. Hum Biol 64, 539-566.

42. Baumgartner RN, Siervogel RM \& Roche AF (1989) Clustering of cardiovascular risk factors in association with indices of adiposity and adipose tissue distribution in adults. Am J Hum Biol 1, 43-52.

43. Huang RC, Mori TA, Burke V, et al. (2009) Synergy between adiposity, insulin resistance, metabolic risk factors, and inflammation in adolescents. Diabetes Care 32, 695-701.

44. Andreeva-Gateva PA, Simeonov VD, Georrgieva-Nikolova RT, et al. (2014) Cluster analysis of the components of the metabolic syndrome in subjects with an increased risk for diabetes mellitus type 2. Endocrinologya 19, 168-185.

45. Ventura AK, Loken E \& Birch LL (2006) Risk profiles for metabolic syndrome in a nonclinical sample of adolescent girls. Pediatrics 118, 2434-2442.

46. Bucci CM, Legnani WE \& Armentano RL (2016) Clinical values dataset processing through cluster analysis to find cardiovascular risk. J Phys Conf Ser 705, 012033.

47. Moazzami AA, Shrestha A, Morrison DA, et al. (2014) Metabolomics reveals differences in postprandial responses to breads and fasting metabolic characteristics associated with postprandial insulin demand in postmenopausal women. J Nutr 144, 807-814.

48. Qureshi W, Wagenknecht L, Watkins S, et al. (2014) Abstract 23: metabolomic features associated with incident hypertension - results from Insulin Resistance Atherosclerosis Study. Circulation 129, Suppl. 1, A23.

49. Krishnan S, Newman JW, Hembrooke TA, et al. (2012) Variation in metabolic responses to meal challenges differing in glycemic index in healthy women: Is it meaningful? Nutr Metab (Lond) 9, 26.

50. Wang TT, Edwards AJ \& Clevidence BA (2013) Strong and weak plasma response to dietary carotenoids identified by cluster analysis and linked to beta-carotene 15,15'-monooxygenase 1 single nucleotide polymorphisms. J Nutr Biochem 24, 1538-1546.

51. Bouwman J, Vogels JT, Wopereis S, et al. (2012) Visualization and identification of health space, based on personalized molecular phenotype and treatment response to relevant underlying biological processes. BMC Med Genomics 5, 1.

52. Chua EC, Shui G, Lee IT, et al. (2013) Extensive diversity in circadian regulation of plasma lipids and evidence for different circadian metabolic phenotypes in humans. Proc Natl Acad Sci U S A 110, 14468-14473.

53. Zák A, Burda M, Vecka M, et al. (2014) Fatty acid composition indicates two types of metabolic syndrome independent of clinical and laboratory parameters. Physiol Res 63, Suppl. 3, S375-S385.

54. Schader LM (2015) Comparison of genetic risk factors between two type II diabetes subtypes. Bachelor Thesis, University of Arizona.

55. Li L, Cheng WY, Glicksberg BS, et al. (2015) Identification of type 2 diabetes subgroups through topological analysis of patient similarity. Sci Transl Med 7, 311ra174.

56. Amato MC, Pizzolanti G, Torregrossa V, et al. (2016) Phenotyping of type 2 diabetes mellitus at onset on the basis of fasting incretin tone: results of a two-step cluster analysis. J Diabetes Investig 7, 219-225.

57. Frei F, Lessa Bde S, Nogueira JC, et al. (2013) Cluster analysis for classification of patients submitted to Fobi-Capella bariatric surgery. Arq Bras Cir Dig 26, Suppl. 1, 33-38.

58. Arguelles W, Llabre MM, Sacco RL, et al. (2015) Characterization of metabolic syndrome among diverse Hispanics/ Latinos living in the United States: latent class analysis from 
the Hispanic Community Health Study/Study of Latinos (HCHS/SOL). Int J Cardiol 184, 373-379.

59. Kim E, Oh W, Pieczkiewicz DS, et al. (2014) Divisive hierarchical clustering towards identifying clinically significant pre-diabetes subpopulations. AMIA Annu Symp Proc 2014, 1815-1824.

60. Mäkinen VP, Soininen P, Forsblom C, et al. (2008) 1H NMR metabonomics approach to the disease continuum of diabetic complications and premature death. Mol Syst Biol 4, 167.

61. Botelho PB, Fioratti CO, Abdalla DS, et al. (2010) Classification of individuals with dyslipidaemia controlled by statins according to plasma biomarkers of oxidative stress using cluster analysis. Br J Nutr 103, 256-265.

62. Holmes E, Loo RL, Stamler J, et al. (2008) Human metabolic phenotype diversity and its association with diet and blood pressure. Nature 453, 396-400.

63. Lenz EM, Bright J, Wilson ID, et al. (2004) Metabonomics, dietary influences and cultural differences: a 1H NMR-based study of urine samples obtained from healthy British and Swedish subjects. I Pharm Biomed Anal 36, 841-849.

64. Dumas ME, Maibaum EC, Teague C, et al. (2006) Assessment of analytical reproducibility of $1 \mathrm{H}$ NMR spectroscopy based metabonomics for large-scale epidemiological research: the INTERMAP Study. Anal Chem 78, 2199-2208.

65. Chaleckis R, Murakami I, Takada J, et al. (2016) Individual variability in human blood metabolites identifies age-related differences. Proc Natl Acad Sci U S A 113, 4252-4259.

66. Bernini $P$, Bertini I, Luchinat $C$, et al. (2009) Individual human phenotypes in metabolic space and time. J Proteome Res $\mathbf{8}$, 4264-4271.

67. Yousri NA, Kastenmüller G, Gieger C, et al. (2014) Long term conservation of human metabolic phenotypes and link to heritability. Metabolomics 10, 1005-1017.

68. Krumsiek J, Mittelstrass K, Do KT, et al. (2015) Genderspecific pathway differences in the human serum metabolome. Metabolomics 11, 1815-1833.

69. Kochhar S, Jacobs DM, Ramadan Z, et al. (2006) Probing genderspecific metabolism differences in humans by nuclear magnetic resonance-based metabonomics. Anal Biochem 352, 274-281.

70. Bartel J, Krumsiek J \& Theis FJ (2013) Statistical methods for the analysis of high-throughput metabolomics data. Comput Struct Biotechnol J 4, e201301009.
71. Valdes AM, Glass D \& Spector TD (2013) Omics technologies and the study of human ageing. Nat Rev Genet 14, 601-607.

72. Kurland IJ, Accili D, Burant C, et al. (2013) Application of combined omics platforms to accelerate biomedical discovery in diabesity. Ann N Y Acad Sci 1287, 1-16.

73. Krug S, Kastenmüller G, Stückler F, et al. (2012) The dynamic range of the human metabolome revealed by challenges. FASEB J 26, 2607-2619.

74. Rezzi S, Martin FP, Alonso C, et al. (2009) Metabotyping of biofluids reveals stress-based differences in gut permeability in healthy individuals. J Proteome Res 8, 4799-4809.

75. Ghini V, Saccenti E, Tenori L, et al. (2015) Allostasis and resilience of the human individual metabolic phenotype. J Proteome Res 14, 2951-2962.

76. Assfalg M, Bertini I, Colangiuli D, et al. (2008) Evidence of different metabolic phenotypes in humans. Proc Natl Acad Sci U S A 105, 1420-1424.

77. Jain AK, Duin RPW \& Mao J (2000) Statistical pattern recognition: a review. IEEE Trans Pattern Anal Mach Intell 22, 4-37.

78. Everitt BS, Landau S, Leese M, et al. (2011) Measurement of proximity. In Cluster Analysis, 5th ed., pp. 67-68 [DJ Balding, NAC Cressie, GM Fitzmaurice, et al., editors]. London: John Wiley \& Sons, Ltd.

79. Kaufman L \& Rousseeuw PJ (2005) Finding Groups in Data: An Introduction to Cluster Analysis. Hoboken: John Wiley \& Sons, Inc.

80. Jain AK, Topchy A, Law MHC, et al. (2004) Landscape of clustering algorithms. Proceedings of the 17th International Conference on Pattern Recognition (ICPR'04), Vol. 1, pp. 260-263.

81. Steinley D (2006) K-means clustering: a half-century synthesis. BrJ Math Stat Psychol 59, 1-34.

82. Bacher J, Pöge A \& Wenzig K (2010) Clusteranalyse - Anwendungsorientierte Einfübrung in Klassifikationsverfabren (Cluster Analysis - Application-Oriented Introduction to Classification Methods), 3rd ed. Munich: Oldenbourg Wissenschaftsverlag $\mathrm{GmbH}$.

83. Dua S \& Chowriappa P (2013) Data Mining for Bioinformatics. Boca Raton, FL: CRC Press Taylor \& Francis Group. 